\title{
Estimativa da área foliar de nabo forrageiro em função de dimensões foliares
}

\author{
Alberto Cargnelutti Filho $\left({ }^{1 *}\right)$; Marcos Toebe $\left({ }^{2}\right)$; Cláudia Burin (3); André Luis Fick $\left({ }^{3}\right)$; Gabriele \\ Casarotto $\left({ }^{2}\right)$ \\ (') Universidade Federal de Santa Maria (UFSM), Centro de Ciências Rurais (CCR), Departamento de Fitotecnia, 97105-900, Santa \\ Maria (RS), Brasil. \\ (2) UFSM, Programa de Pós-Graduação em Agronomia, Santa Maria (RS), Brasil. \\ (3) UFSM, Curso de Graduação em Engenharia Florestal, Santa Maria (RS), Brasil. \\ (*) Autor correspondente: alberto.cargnelutti.filho@gmail.com
}

Recebido: 23/set./2011; Aceito: 27/dez./2011

\begin{abstract}
Resumo
O objetivo deste trabalho foi desenvolver um modelo para estimar a área foliar de nabo forrageiro (Raphanus sativus L. var. oleiferus Metzg) determinada por fotos digitais, em função do comprimento, ou da largura e/ou do produto comprimento vezes largura da folha. Aos 76 dias após a semeadura, foram coletadas 557 folhas da haste principal de 92 plantas, sendo mensurados o comprimento $(\mathrm{C})$ e a largura $(\mathrm{L})$ de cada folha, e calculado o produto comprimento $\times \operatorname{largura}(\mathrm{C} \times \mathrm{L})$. Após, determinou-se a área foliar $(Y)$, por meio do método de fotos digitais. Do total de folhas, separaram-se, aleatoriamente, 450 folhas para a construção de modelos do tipo quadrático, potência e linear de $Y$ em função de $C$, da L, e/ou de CxL. 107 folhas foram usadas para a validação dos modelos. O modelo do tipo potência da área foliar obtida por meio do método de fotos digitais ( $\left.\hat{Y}=0,6843 x^{0,9221}, R^{2}=0,9862\right)$ em função do produto comprimento $\times$ largura é adequado para estimar a área foliar de nabo forrageiro.
\end{abstract}

Palavras-chave: Raphanus sativus L. var. oleiferus Metzg, fotos digitais, modelagem, método não destrutivo.

\section{Estimate of leaf area of forage turnip according to leaf dimensions}

\section{Abstract}

The objective of this work was build a model to estimate leaf area of forage turnips (Raphanus sativus L. var. oleiferus Metzg) determined by digital photos with the length or width and/or the product length $\times$ width of the leaf. Seventy-six days after sowing of forage turnip, 557 leaves were collected in the main stem of 92 plants. The length (C) and width (L) of each leaf were measured and the product length $\times$ width $(C \times L)$ calculated. The leaf area $(Y)$ was determined by the method of digital photos. From the total of leaves, 450 leaves were randomly separated to build models of the types quadratic, potency and linear with $Y$ as function of $C, L$ and/or $C \times L$. 107 leaves were used for model validation. The potency model of the leaf area obtained by the method of digital photos $\left(\hat{Y}=0.6843 x^{0.9221}, R^{2}=0.9862\right)$ based on the product length $\times$ width is adequate to estimate the leaf area in forage turnip.

Key words: Raphanus sativus L. var. oleiferus Metzg, digital photos, modeling, non destructive method.

O nabo forrageiro (Raphanus sativus L. var. oleiferus Metzg) é uma planta anual, herbácea, ereta e ramificada, pertencente à família Brassicaceae (Derpsch e Calegari, 1992). É importante no processo de rotaçáo de culturas, devido à sua capacidade de ciclagem de nutrientes e de produçáo de massa verde. Essa cultura tem alta taxa de decomposiçáo da palhada e é de rápida liberação de nutrientes para as culturas sucessoras (Ceretta et al., 2002). Nesse contexto, Crusciol et al. (2005) verificaram que a quantidade de nutrientes liberada até o estádio de pré-florescimento é elevada, com acúmulo aproximado de $57,2 \mathrm{~kg} \mathrm{ha}^{-1}$ de N, 15,3 $\mathrm{kg} \mathrm{ha}^{-1}$ de $\mathrm{P}, 85,7 \mathrm{~kg} \mathrm{ha}^{-1}$ de $\mathrm{K}, 37,4 \mathrm{~kg} \mathrm{ha}^{-1}$ de Ca, $12,5 \mathrm{~kg}$ $\mathrm{ha}^{-1}$ de $\mathrm{Mg}$ e $14,0 \mathrm{~kg} \mathrm{ha}^{-1}$ de $\mathrm{S}$ e produção média de $2.938 \mathrm{~kg} \mathrm{ha}^{-1}$ de massa seca de parte aérea.

A área foliar é importante na mensuração do crescimento de uma cultura, e pode ser determinada diretamente por meio do método de fotos digitais. $\mathrm{Na}$ cultura do cafeeiro, Tavares Júnior et al. (2002) verificaram que o processamento de imagens digitais é considerado adequado e exato para a estimativa da área foliar em substituição ao método que utiliza o aparelho medidor de área foliar LI-COR ${ }^{\circledR}$ (método padrão) (Li-cor 3100, 1996). Ainda, Adami et al. (2008) constataram que o método de fotos digitais pode substituir o método LI-COR ${ }^{\circledR}$, em folíolos 
íntegros e danificados de soja, com custo inferior e acurácia similar.

Indiretamente, a área foliar pode ser obtida por modelos de estimativa de área foliar em função de dimensões lineares das folhas, sendo útil quando as plantas não podem ser destruídas para que a medição direta seja realizada (BLANCO e Folegatti, 2005). Modelos que relacionam a área foliar com dimensóes lineares das folhas foram desenvolvidos para diversas plantas, como pepineiro (Blanco e Folegatti, 2005), Sida cordifolia e Sida rhombifolia (BIANCO et al., 2008), Curcuma alismatifolia e Curcuma zedoaria (РinTo et al., 2008), girassol (Maldaner et al., 2009) e crambe (Toebe et al., 2010), entre outras. No entanto, esses modelos para nabo forrageiro não foram encontrados na literatura. Assim, o objetivo deste trabalho foi desenvolver um modelo para estimar a área foliar de nabo forrageiro (Raphanus sativus L. var. oleiferus Metzg) determinada por fotos digitais, em funçáo do comprimento, ou da largura e/ou do produto comprimento vezes largura da folha.

Foi realizado um experimento com a cultura de nabo forrageiro em uma área experimental de tamanho $12 \mathrm{~m} \mathrm{x}$ $12 \mathrm{~m}\left(144 \mathrm{~m}^{2}\right)$. A semeadura foi realizada em 10/6/2010 e o espaçamento utilizado foi de $0,2 \mathrm{~m}$ entre fileiras e $0,0667 \mathrm{~m}$ entre plantas na fileira, totalizando 75 plantas por $\mathrm{m}^{2}$. Na análise química do solo na camada de $0-20 \mathrm{~cm}$ foram avaliados os seguintes atributos: teor de argila, $290 \mathrm{~g} \mathrm{~kg}^{-1} ; \mathrm{pH}$ em água, 5,3; teor de matéria orgânica, $21 \mathrm{~g} \mathrm{dm}^{-3}$; Al trocá-

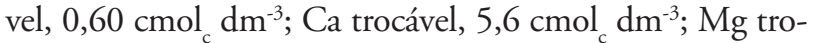
cável, $2,0 \mathrm{cmol}_{\mathrm{c}} \mathrm{dm}^{-3}$; P disponível, $13,5 \mathrm{mg} \mathrm{dm}^{-3}$ e K trocável, $144 \mathrm{mg} \mathrm{dm}^{-3}$; índice SMP, 5,7 e saturação de bases, $56 \%$ (CQFSRS/SC, 2004). A adubação de base foi de 30 $\mathrm{kg} \mathrm{ha}^{-1}$ de N, $150 \mathrm{~kg} \mathrm{ha}^{-1}$ de $\mathrm{P}_{2} \mathrm{O}_{5}$ e $150 \mathrm{~kg} \mathrm{ha}^{-1}$ de $\mathrm{K}_{2} \mathrm{O}$ e a adubaçáo de cobertura, de $100 \mathrm{~kg}$ de $\mathrm{N} \mathrm{ha}^{-1}$, aplicada aos 28 dias após a emergência. Aos 76 dias após a emergência, quando as plantas estavam em pleno florescimento, foram coletadas, aleatoriamente, 92 plantas. Dessas plantas, foram retiradas as folhas da haste principal para a determinação da área foliar, totalizando 557 folhas.

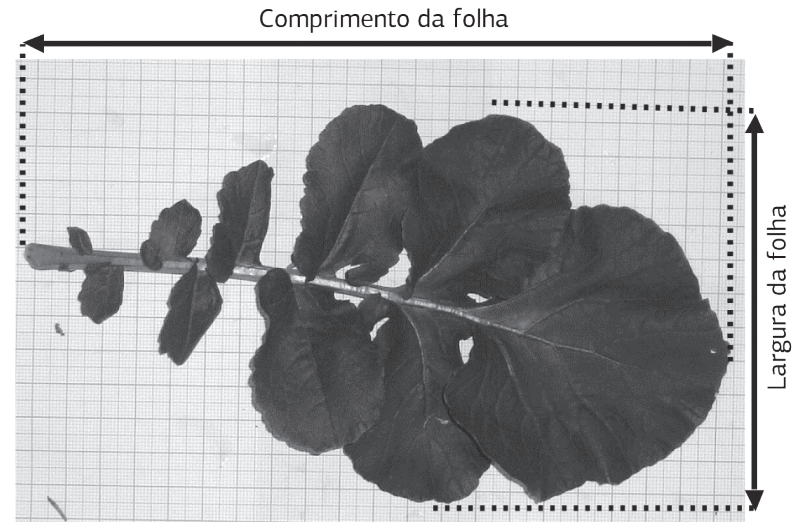

Figura 1. Comprimento e largura de uma folha de nabo forrageiro (Raphanus sativus L. var. oleiferus Metzg).
Nessas 557 folhas foram mensurados o comprimento da folha $(\mathrm{C}$, em $\mathrm{cm})$, a partir da inserção na haste principal, e a largura da folha $(\mathrm{L}, \mathrm{em} \mathrm{cm})$, determinada pela máxima largura medida perpendicularmente à nervura central, com régua milimetrada (Figura 1). Após, foi calculado o produto comprimento vezes largura da folha $\left(\mathrm{C} \times \mathrm{L}, \mathrm{em} \mathrm{cm}^{2}\right)$. A seguir, as mesmas folhas foram colocadas em cima de papel milimetrado e fotografadas por meio de uma câmera digital da marca Sony, modelo DSC-W110. Essas imagens (fotos) foram processadas com o programa Sigma Scan Pro v. 5.0, Jandel Scientific (JANDEL SCIENTIFIC, 1991), para a determinação da área foliar por meio do método de fotos digitais $\left(\mathrm{Y}, \mathrm{em} \mathrm{cm}^{2}\right)$.

Posteriormente, dentre as 557 folhas foram selecionadas, aleatoriamente, 107 folhas, utilizadas somente na validação dos modelos. Com os dados de $\mathrm{C}, \mathrm{L}, \mathrm{C} \times \mathrm{L}$ e de Y das 450 folhas restantes, foram feitos histogramas de frequência e diagramas de dispersão e calculadas as medidas de tendência central e de variabilidade. Depois, modelou-se a área foliar $(\mathrm{Y})$ em função do $\mathrm{C}$, ou da L e/ou do $\mathrm{C} \times \mathrm{L}$ da folha, por meio dos modelos: quadrático $\left(\mathrm{Y}=\mathrm{a}+\mathrm{bx}+\mathrm{cx}^{2}\right)$, potência $\left(\mathrm{Y}=\mathrm{ax} \mathrm{x}^{\mathrm{b}}\right)$ e linear $(\mathrm{Y}=\mathrm{a}+\mathrm{bx}$ ), totalizando nove modelos (três tipos de modelos $\times$ três variáveis independentes).

A validação dos nove modelos de estimativa de área foliar foi realizada com base nos 107 valores estimados pelo modelo $\left(\hat{Y}_{\mathrm{i}}\right)$ e os 107 valores observados $\left(\mathrm{Y}_{\mathrm{i}}\right)$. Em cada modelo, inicialmente, foi ajustada uma regressão linear simples $\left(\hat{Y}_{i}=a+b Y_{i}\right)$ da área foliar estimada pelo modelo (variável dependente) em função da área foliar determinada pelo método de fotos digitais (variável independente). Foram testadas as hipóteses $\mathrm{H}_{0}: \mathrm{a}=0$ versus $\mathrm{H}_{1}: \mathrm{a} \neq 0$ e $\mathrm{H}_{0}: \mathrm{b}=1$ versus $\mathrm{H}_{1}: \mathrm{b} \neq 1$, por meio do teste $t$ de Student a $5 \%$ de probabilidade de erro. A seguir foram calculados os coeficientes de correlação linear de Pearson $(r)$ e de determinação $\left(R^{2}\right)$ entre $\hat{Y}_{i}$ e $Y_{i}$. Para cada modelo, foi calculado o erro absoluto médio (EAM), a raiz do quadrado médio do erro (RQME) e o índice d de Willmott (Willmotт, 1981), por meio, respectivamente, das expressóes:

$$
\begin{aligned}
& \operatorname{EAM}=\frac{\sum_{\mathrm{i}=1}^{\mathrm{n}}\left|\hat{\mathrm{Y}}_{\mathrm{i}}-\mathrm{Y}_{\mathrm{i}}\right|}{\mathrm{n}}, \operatorname{RQME}=\sqrt{\frac{\sum_{\mathrm{i}=1}^{\mathrm{n}}\left(\hat{\mathrm{Y}}_{\mathrm{i}}-\mathrm{Y}_{\mathrm{i}}\right)^{2}}{\mathrm{n}}} \mathrm{e} \\
& \mathrm{d}=1-\left[\frac{\sum_{\mathrm{i}=1}^{\mathrm{n}}\left(\hat{\mathrm{Y}}_{\mathrm{i}}-\mathrm{Y}_{\mathrm{i}}\right)^{2}}{\sum_{\mathrm{i}=1}^{\mathrm{n}}\left(\left|\hat{Y}_{\mathrm{i}}-\overline{\mathrm{Y}}\right|+\left|\mathrm{Y}_{\mathrm{i}}-\overline{\mathrm{Y}}\right|\right)^{2}}\right]
\end{aligned}
$$

em que: $\hat{Y}_{\mathrm{i}}$ são os valores estimados de área foliar, $\mathrm{Y}_{\mathrm{i}}$ são os valores observados de área foliar por meio do método de fotos digitais, $\bar{Y}$ é a média dos valores observados e n é o número de folhas ( $\mathrm{n}=107)$. Após obteve-se o índice CS de 
Camargo e Sentelhas (1997) pelo produto entre $\mathrm{r}$ e d $(\mathrm{CS}=\mathrm{r} \times \mathrm{d})$. Esse índice é adequado para indicar o desempenho dos métodos e segundo Camargo e Sentelhas (1997) o "r" representa a precisão e o "d" a exatidão.

Os critérios utilizados para a seleção dos modelos que melhor estimam a área foliar de nabo forrageiro em função do $\mathrm{C}$, ou da $\mathrm{L}$ e/ou do $\mathrm{C} \times \mathrm{L}$ foram: coeficiente linear (a) não diferente de zero, coeficiente angular (b) não diferente de um, coeficientes de correlaçáo linear de Pearson $(\mathrm{r})$ e de determinação $\left(\mathrm{R}^{2}\right)$ mais próximos de um, erro absoluto médio (EAM) e raiz do quadrado médio do erro (RQME) mais próximo de zero, e índices d e CS mais próximos de um. As análises estatísticas foram realizadas com auxílio do aplicativo Microsoft Office Excel e do software Statistica 7.0 (Statsoft, 2005).

A ampla variabilidade do tamanho das folhas, verificada pela amplitude do comprimento, da largura e da área foliar, aliada à elevada magnitude do coeficiente de variação (Tabela 1), é importante para a geração de modelos, pois permite sua utilização em folhas pequenas, médias e grandes. Portanto, esse amplo conjunto de dados de comprimento (C), largura (L), produto comprimento vezes largura $(\mathrm{C} \times \mathrm{L})$ e área foliar $(\mathrm{Y})$ determinada por meio de fotos digitais (450 folhas) é adequado para o estudo proposto.

Os diagramas de dispersão, entre as variáveis C, $\mathrm{L}, \mathrm{C} \times \mathrm{L}$ e $\mathrm{Y}$, revelam padróes nos dados que sugerem o ajuste de modelos não lineares e lineares (Figura 2). De maneira geral, os modelos quadrático, potência e linear que relacionam a área foliar determinada por meio de fotos digitais com o comprimento, ou com a largura e/ ou com o produto comprimento vezes largura, apresentaram elevados valores do coeficiente de determinação $\left(\mathrm{R}^{2} \geq 0,8939\right)$ (Tabela 2). Os modelos quadrático, potência e linear ajustados em função do produto comprimento vezes largura tiveram desempenhos superiores $\left(R^{2} \geq 0,9469\right)$ aos ajustados em função do comprimento

Tabela 1. Mínimo, máximo, amplitude, média, mediana e coeficiente de variaçáo (CV), do comprimento (C), da largura (L), do produto comprimento vezes largura $(\mathrm{C} \times \mathrm{L})$ e da área foliar determinada por fotos digitais (Y) de 450 folhas de nabo forrageiro (Raphanus sativus L. var. oleiferus Metzg)

\begin{tabular}{lrrrr} 
Estatística & $\mathbf{C}(\mathbf{c m})$ & $\mathbf{L}(\mathbf{c m})$ & $\mathbf{C} \times \mathbf{L}\left(\mathbf{c m}^{2}\right)$ & $\mathbf{Y}\left(\mathbf{c m}^{2}\right)$ \\
\hline Mínimo & 3,5000 & 1,8000 & 9,7200 & 4,6851 \\
\hline Máximo & 49,1000 & 18,8000 & 885,4800 & 317,0835 \\
Amplitude & 45,6000 & 17,0000 & 875,7600 & 312,3984 \\
Média & 19,8704 & 9,7322 & 234,2578 & 101,4652 \\
Mediana & 18,3000 & 10,6000 & 200,7300 & 97,3039 \\
CV (\%) & 56,4232 & 41,9008 & 80,5555 & 72,7374
\end{tabular}
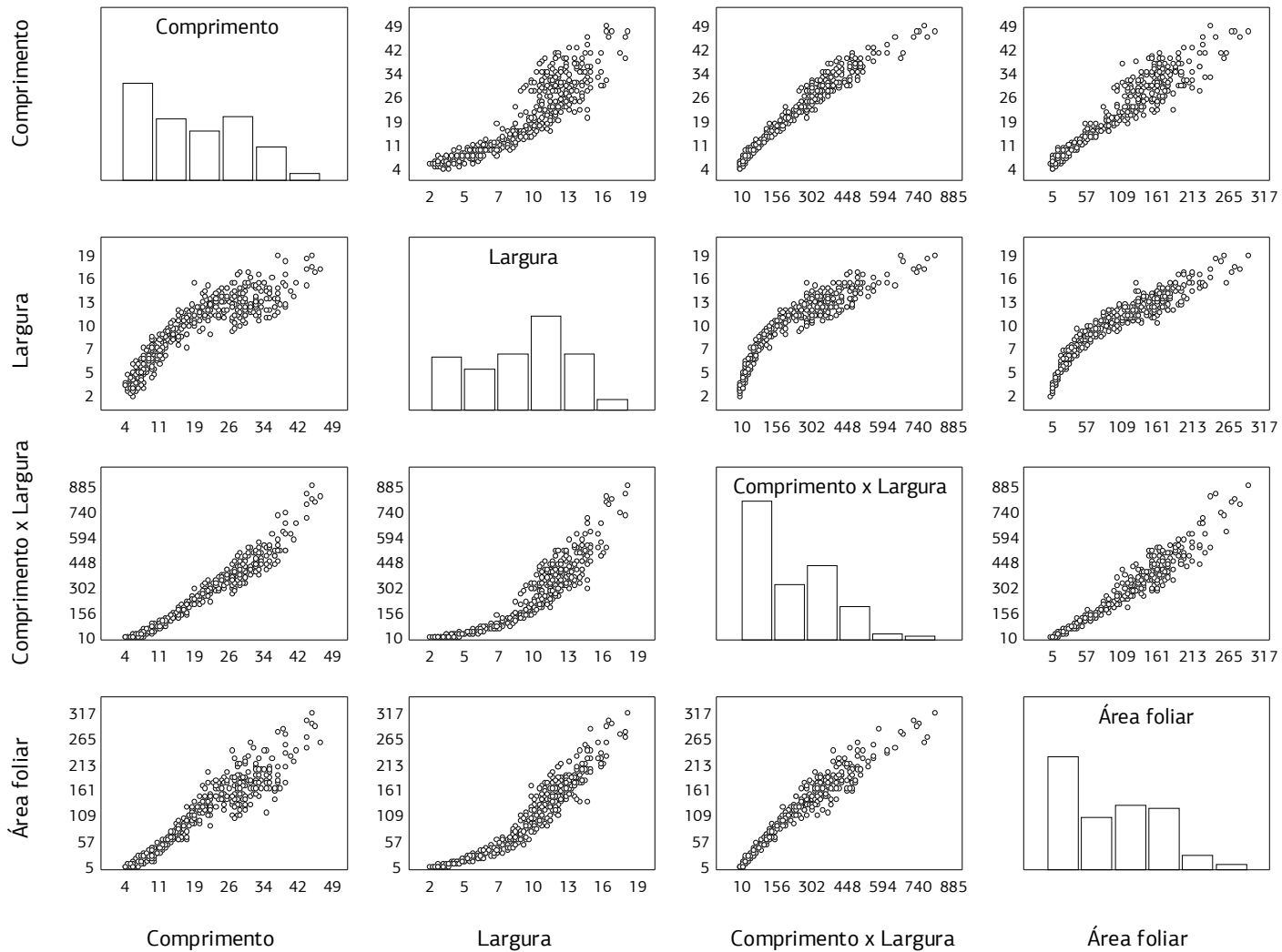

Figura 2. Matriz com o histograma de frequência (na diagonal) e gráficos de dispersão entre o comprimento, em cm, a largura, em cm, o produto comprimento $\mathrm{x}$ largura, em $\mathrm{cm}^{2} \mathrm{e}$ a área foliar determinada por fotos digitais, em $\mathrm{cm}^{2}$ de 450 folhas de nabo forrageiro (Raphanus sativus L. var. oleiferus Metzg). 
ou da largura. Desempenhos superiores de modelos ajustados em funçáo do produto comprimento vezes largura também foram encontrados nas plantas de pepino (Blanco e Folegatti, 2005), de Sida cordifolia e Sida rhombifolia (BIANco et al., 2008) e de Curcuma alismatifolia e Curcuma zedoaria (PInTo et al., 2008).

Entre os tipos de modelos, potência obteve os maiores valores de coeficientes de determinação (melhor ajuste) das estimativas de área foliar, concordando com MaLdANER et al. (2009) ao afirmarem que esse modelo é o mais adequado para a estimativa de área foliar de girassol. Logo, o modelo tipo potência da área foliar obtida por meio do método de fotos digitais em função do produto comprimento vezes largura $\left(\hat{\mathrm{Y}}=0,6843 \mathrm{x}^{0,9221}\right.$, $\mathrm{R}^{2}=0,9862$ ), é adequado para estimar a área foliar de nabo forrageiro (Tabela 2 e Figuras 3 e 4). Esse modelo proporcionou coeficiente linear náo diferente de zero e coeficiente angular náo diferente de um. Além disso, comparado aos demais oito modelos, resultou nos maiores valores de coeficientes de correlação linear de Pearson (r) e de determinação $\left(\mathrm{R}^{2}\right)$, os menores valores de erro absoluto médio (EAM) e raiz do quadrado médio do erro (RQME) e os maiores índices d e CS (Tabela 3). Portanto, os indicadores de validação confirmaram que esse é o modelo mais preciso.

$\mathrm{Na}$ prática, a medição do comprimento e da largura da folha é suficiente para estimar a área foliar que seria determinada por meio do método de fotos digitais. Esse resultado é importante, pois possibilita determinar a área foliar, com precisão, durante todo o ciclo da cultura, sem a necessidade de destruição das folhas. O modelo de potência da área foliar obtida por meio do método de fotos digitais em função do produto comprimento vezes largura $\left(\hat{\mathrm{Y}}=0,6843 \mathrm{x}^{0,9221}\right)$, é adequado para estimar a área foliar de nabo forrageiro (Raphanus sativus L. var. oleiferus Metzg).

Tabela 2. Equações para a determinação da área foliar por fotos digitais (Y), utilizando o comprimento (C), a largura (L) e o produto comprimento vezes largura $(\mathrm{C} \times \mathrm{L})$ como variáveis independentes $(\mathrm{x})$ e coeficiente de determinação, com base em 450 folhas de nabo forrageiro (Raphanus sativus L. var. oleiferus Metzg)

\begin{tabular}{|c|c|c|c|}
\hline Modelo & Variável independente $(x)$ & Equação & Coeficiente de determinação \\
\hline 1) Quadrático & C & $\hat{Y}=-37,7200+8,1036 x-0,0420 x^{2}$ & 0,9113 \\
\hline 2) Quadrático & L & $\hat{Y}=-6,5444+1,1887 x+0,8664 x^{2}$ & 0,9305 \\
\hline 3) Quadrático & $C \times L$ & $\hat{Y}=1,7165+0,5069 x-0,0002 x^{2}$ & 0,9616 \\
\hline 4) Potência & C & $\hat{Y}=0,7371 x^{1,6059}$ & 0,9383 \\
\hline 5) Potência & L & $\hat{Y}=0,8985 x^{1,9926}$ & 0,9669 \\
\hline 6) Potência & $C \times L$ & $\hat{Y}=0,6843 x^{0,9221}$ & 0,9862 \\
\hline 7) Linear & C & $\hat{Y}=-23,0923+6,2685 x$ & 0,9068 \\
\hline 8) Linear & L & $\hat{Y}=-65,0636+17,1111 x$ & 0,8939 \\
\hline 9) Linear & $C \times L$ & $\hat{Y}=12,3117+0,3806 x$ & 0,9469 \\
\hline
\end{tabular}

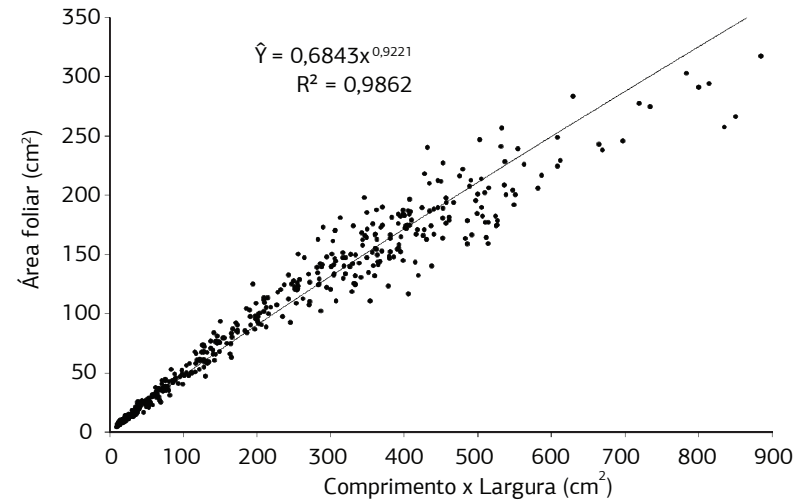

Figura 3. Modelo tipo potência, da área foliar determinada por fotos digitais $\left(\mathrm{em} \mathrm{cm}^{2}\right)$ em função do produto comprimento $\mathrm{x}$ largura $\left(\mathrm{em} \mathrm{cm}^{2}\right)$, com base em 450 folhas de nabo forrageiro (Raphanus sativus L. var. oleiferus Metzg).

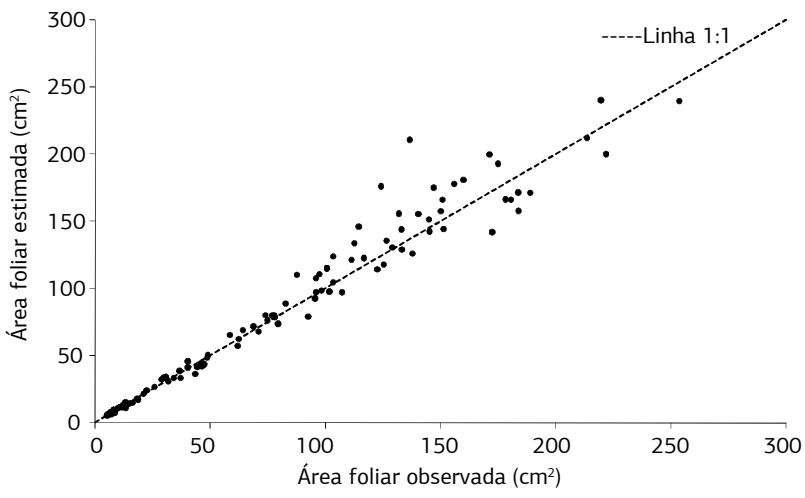

Figura 4. Relaçáo entre os valores de área foliar observada (determinada) por meio do método de fotos digitais e a área foliar estimada em função do comprimento x largura, através do modelo tipo potência, em 107 folhas de nabo forrageiro (Raphanus sativus L. var. oleiferus Metzg). 
Tabela 3. Variáveis independentes (x) e coeficientes linear (a), angular (b), de correlação linear de Pearson (r) e de determinação ( $\left.\mathrm{R}^{2}\right)$, obtidos na regressão linear ajustada entre a área foliar estimada (variável dependente) e a observada (variável independente). Erro absoluto médio (EAM), raiz do quadrado médio do erro (RQME), índice d de Willmott (Willmott, 1981) e CS de Camargo e Sentelhas (1997) calculados com base nas áreas foliares estimada e observada, de 107 folhas de nabo forrageiro (Raphanus sativus L. var. oleiferus Metzg)

\begin{tabular}{|c|c|c|c|c|c|c|c|c|c|}
\hline Modelo & $x^{(1)}$ & $a^{(2)}$ & $b^{(3)}$ & $r^{(4)}$ & $\mathbf{R}^{2}$ & EAM & RQME & d & CS \\
\hline 1) Quadrático & C & $6,0922^{*}$ & $1,0068^{\text {ns }}$ & $0,9586^{*}$ & 0,9190 & 14,0866 & 19,6776 & 0,9756 & 0,9353 \\
\hline 2) Quadrático & L & $9,1412^{*}$ & $0,9174^{*}$ & $0,9629^{*}$ & 0,9273 & 12,3557 & 16,9013 & 0,9801 & 0,9439 \\
\hline 3) Quadrático & $\mathrm{C} \times \mathrm{L}$ & $4,7429^{*}$ & $0,9953^{\text {ns }}$ & $0,9780^{*}$ & 0,9565 & 8,7435 & 13,8602 & 0,9875 & 0,9659 \\
\hline 4) Potência & C & $-2,1776^{\mathrm{ns}}$ & $1,0808^{*}$ & $0,9455^{*}$ & 0,8941 & 14,4277 & 23,9933 & 0,9665 & 0,9139 \\
\hline 5) Potência & L & $11,0979^{*}$ & $0,8635^{*}$ & $0,9642^{*}$ & 0,9298 & 11,6993 & 16,9646 & 0,9788 & 0,9438 \\
\hline 6) Potência & $C \times L$ & $1,5817^{\text {ns }}$ & $1,0180^{\text {ns }}$ & $0,9782^{*}$ & 0,9569 & 8,1068 & 13,7651 & 0,9880 & 0,9665 \\
\hline 7) Linear & C & $8,3797^{*}$ & $0,9731^{\text {ns }}$ & $0,9588^{*}$ & 0,9194 & 13,0516 & 18,9819 & 0,9764 & 0,9363 \\
\hline 8) Linear & L & $6,2238^{\text {ns }}$ & $0,9773^{\text {ns }}$ & $0,9296^{*}$ & 0,8642 & 20,5156 & 24,4493 & 0,9619 & 0,8942 \\
\hline 9) Linear & $C \times L$ & $9,8579^{*}$ & $0,9217^{*}$ & $0,9771^{*}$ & 0,9547 & 10,1069 & 13,8098 & 0,9867 & 0,9641 \\
\hline
\end{tabular}

(1) C: comprimento, L: largura e $\mathrm{C} \times \mathrm{L}$ : comprimento vezes largura de folhas.

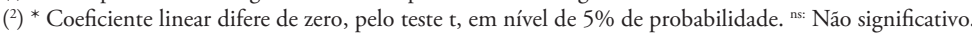

$\left({ }^{3}\right) *$ Coeficiente angular difere de um, pelo teste t, em nível de $5 \%$ de probabilidade. ns: Não significativo.

$\left({ }^{4}\right) *$ Coeficiente de correlação difere de zero, pelo teste $\mathrm{t}$, em nível de $5 \%$ de probabilidade.

\section{REFERÊNCIAS}

ADAMI, M.; HASTENREITER, F.A.; FLUMIGNAN, D.L.; FARIA, R.T. Estimativa de área de folíolos de soja usando imagens digitais e dimensóes foliares. Bragantia, v.67, p.1053-1058, 2008.

BLANCO, F.F.; FOLEGATTI, M.V. Estimation of leaf area for greenhouse cucumber by linear measurements under salinity and grafting. Scientia Agricola, v.62, p.305-309, 2005.

BIANCO, S.; CARVALHO, L.B.; BIANCO, M.S. Estimativa da área foliar de Sida cordifolia e Sida rhombifolia usando dimensôes lineares do limbo foliar. Planta Daninha, v.26, p.807-813, 2008.

CAMARGO, A.P.; SENTELHAS, P.C. Avaliação do desempenho de diferentes métodos de estimativa da evapotranspiraçáo potencial no estado de São Paulo, Brasil. Revista Brasileira de Agrometeorologia, v.5, p.89-97, 1997.

CERETTA, C.A.; BASSO, C.J.; HERBES, M.G.; POLETTO, N.; SILVEIRA, M.J. Produção e decomposição de fitomassa de plantas invernais de cobertura de solo e milho, sob diferentes manejos da adubação nitrogenada. Ciência Rural, v.32, p.49-54, 2002.

COMISSÃO DE QUÍMICA E FERTILIDADE DE SOLO DO RIO GRANDE DO SUL E DE SANTA CATARINA - CQFSRS/ SC. Recomendações de adubação e de calagem para os Estados do RS e SC. 3.ed. Passo Fundo: SBCS-Núcleo Regional Sul, 2004. 400p.

CRUSCIOL, C.A.C.; COTTICA, R.L.; LIMA, E.V.; ANDREOTTI, M.; MORO, E.; MARCON, E. Persistência de palhada e liberação de nutrientes do nabo forrageiro no plantio direto. Pesquisa Agropecuária Brasileira, v.40, p.161-168, 2005.

DERPSCH, R.; CALEGARI, A. Plantas para adubaçáo verde de inverno. Londrina: IAPAR, 1992. 80p. (Circular, 73)

JANDEL SCIENTIFIC. User's Manual. California, 1991. 280p.

LI-COR ${ }^{\circledR}$. LI 3100 area meter instruction manual. Lincoln: $\mathrm{LICOR}^{\circledR}, 1996.34 \mathrm{p}$.

MALDANER, I.C.; HELDWEIN, A.B.; LOOSE, L.H.; LUCAS, D.D.P.; GUSE, F.I.; BORTOLUZZI, M.P. Modelos de determinação não-destrutiva da área foliar em girassol. Ciência Rural, v.39, p.1356-1361, 2009.

PINTO, A.C.R.; GRAZIANO, T.T.; BARBOSA, J.C.; LASMAR, F.B. Modelos para estimativa da área foliar de Curcuma alismatifolia e Curcuma zedoaria. Bragantia, v.67, p.549-552, 2008.

STATSOFT. Statistica 7.0 Software. Tucksa, USA, 2005.

TAVARES JUNIOR, J.E.; FAVARIN, J.L.; DOURADO NETO, D.; MAIA, A.H.N.; FAZUOLI, L.C.; BERNARDES, M.S. Análise comparativa de métodos de estimativa de área foliar em cafeeiro. Bragantia, v.61, p.199-203, 2002.

TOEBE, M.; BRUM, B.; LOPES, S.J.; CARGNELUTTI FILHO, A.; SILVEIRA, T.R. Estimativa da área foliar de Crambe abyssinica por discos foliares e por fotos digitais. Ciência Rural, v.40, p.445448, 2010.

WILLMOTT, C.J. On the validation of models. Physical Geography, v.2, p.184-194, 1981. 